\title{
Magnetic pattern at supergranulation scale: the void size distribution
}

\author{
F. Berrilli, S. Scardigli, and D. Del Moro
}

Department of Physics, University of Rome Tor Vergata, 00133, Rome, Italy

e-mail: berrilli@roma2.infn.it

Received 17 April 2014 / Accepted 2 July 2014

\begin{abstract}
The large-scale magnetic pattern observed in the photosphere of the quiet Sun is dominated by the magnetic network. This network, created by photospheric magnetic fields swept into convective downflows, delineates the boundaries of large-scale cells of overturning plasma and exhibits "voids" in magnetic organization. These voids include internetwork fields, which are mixed-polarity sparse magnetic fields that populate the inner part of network cells. To single out voids and to quantify their intrinsic pattern we applied a fast circle-packing-based algorithm to $511 \mathrm{SOHO} / \mathrm{MDI}$ high-resolution magnetograms acquired during the unusually long solar activity minimum between cycles 23 and 24 . The computed void distribution function shows a quasi-exponential decay behavior in the range 10-60 Mm. The lack of distinct flow scales in this range corroborates the hypothesis of multi-scale motion flows at the solar surface. In addition to the quasi-exponential decay, we have found that the voids depart from a simple exponential decay at about $35 \mathrm{Mm}$.
\end{abstract}

Key words. Sun: magnetic fields - Sun: photosphere - convection

\section{Introduction}

The nature of the multiscale magnetic pattern observed on the solar surface remains a long-standing puzzle in solar physics. The instability responsible for the solar convective zone is due to the increase of opacity in the outer envelope of the star. The resulting turbulent convection creates temperature and velocity structures that evolve over a range of spatial and temporal multiple scales. Large-scale plasma motions (i.e., differential rotation, meridional circulation, and torsional oscillations) and the solar magnetic field contribute to increase the complexity of both the solar surface dynamics and its appearence. Since the convective plasma flows govern the motion of the single magnetic features, analyzing the photospheric magnetic pattern provides a way to investigate all the organization scales of convection, from granulation to the global circulation (e.g., Yelles Chaouche et al. 2011).

Three different spatial and temporal scales are traditionally identified on the solar surface: the granulation $(\sim 1 \mathrm{Mm}$ wide, lifetimes of a few minutes), the meso-granulation $(5-10 \mathrm{Mm}$, lifetimes of a few hours), and the supergranulation (30-50 Mm, lifetimes of about one day). As reported by Nordlund et al. (2009), a division defined like this is probably of historical and not physical origin. Indeed, all the analyses of the photospheric motion spectrum indicate that it covers all scales, from the global down to the subgranular. Different image sampling and identification techniques, using different windows in space and time domains, may be responsible for the perceived different spatial patterns and different dominant scales.

In more detail, from the 1960 s on (Leighton et al. 1962; Leighton 1964), supergranulation has been the subject of a number of studies focused on its origin (Rast 2003; Crouch et al. 2007; Stein et al. 2007), its spatial scale (Schrijver et al. 1997; Berrilli et al. 1998; Del Moro et al. 2004; Meunier et al. 2008), its structure and evolution (DeRosa \& Toomre 2004; Del Moro et al. 2007; Goldbaum et al. 2009; Duvall \& Birch 2010), its large-scale organization (Berrilli et al. 2004, 2005; Lisle et al. 2004), and its relationship with the surface magnetic fields (Lisle et al. 2000; Rieutord et al. 2000; Orozco Suárez et al. 2012; Giannattasio et al. 2014), to cite just a few. A detailed review of these efforts is presented in Rieutord \& Rincon (2010).

Although some differences between the supergranulation pattern and the magnetic network have been reported, (e.g. Rieutord \& Rincon 2010), the general conclusion is that convective cells at supergranular scale are outlined by the magnetic network, which is a large-scale interconnected pattern of magnetic elements. With increasing spatial resolution of instrumentation during the past few years, it has been made clear that the so-called quiet Sun is in fact crowded with magnetic-flux structures. Their magnetic field strengths range from less than 200 to 1000 Gauss.

The inspection of photospheric magnetograms that were taken at the limits of the available resolution, (Sánchez Almeida 2003; Lites et al. 2008; Martìnez González et al. 2012; Orozco Suárez \& Bellot Rubio 2012) reveals regions where magnetic fields are weak and very inclined, commonly named voids, whose spatial distribution and pattern is generated by underlying plasma flow structures.

In this paper we study the void size distribution (VSD) to determine whether it reveals distinct flow scales (e.g., supergranular scale) or it is smoothly distributed. The automatic and accurate identification of voids, which is necessary to quantify their geometrical properties, is performed using an improved version of the void-detection algorithm introduced in Berrilli et al. (2013; henceforth Paper I). A novel and faster circlepacking algorithm has been developed and applied to identify such voids in the 10-60 Mm spatial range of SOHO/MDI highresolution quiet-Sun magnetograms acquired during the past 
unusual minimum in solar activity. We stress that the analysis of void statistics, as derived from the VSD could be a powerful tool for describing the magnetic pattern observed in the photosphere and maybe for testing current and future solar MHD numerical simulations.

\section{Dataset}

The analysis was performed on an extended series of SOHO/MDI magnetograms acquired during the solar activity minimum between cycles 23 and 24. The Solar Oscillations Investigation/Michelson Doppler Imager (SOI/MDI) instrument (Scherrer et al. 1995) onboard the SOHO spacecraft acquired images of the Sun from 1996 to 2011 using the spectral line Ni I $676.78 \mathrm{~nm}$ (Liu \& Norton 2001; Criscuoli et al. 2011). The images were recorded by a $1024 \times 1024$ CCD camera in two spatial resolution modes: full-disk and high-resolution of the central part of the disk (HR).

We have analyzed a dataset of 511 high-resolution magnetograms selected to cover a period of 18 months from Jan. 1, 2008 to Jun. 30, 2009. We carried out our analysis on the level 1.8 high-resolution magnetograms and excluded from our dataset a few images that showed strong magnetic activity. The images have a field of view (FOV) of $11^{\prime} \times 11^{\prime}$ with a plate scale of $0.625^{\prime \prime}$ per pixel and a (diffraction-limited) resolution of $1.25^{\prime \prime}$.

These magnetograms measure the total amount of the longitudinal component of the magnetic flux density in each single resolution element: $\langle|\boldsymbol{B}| \cos \gamma\rangle$, averaged over the pixel, where $\gamma$ is the angle between the magnetic vector $\boldsymbol{B}$ and the line of sight. In this work, we assumed the LOS component of the magnetic field to be normal to the solar surface. We hereafter refer to $|\boldsymbol{B}|<\cos \gamma>$ as $B$.

Figure 1 shows the histogram of the magnetic signal that covers the whole dataset: its core is approximated clearly by a Gaussian distribution (as in, e.g., Krivova \& Solanki 2004). The standard deviation of the magnetic signal measured from our dataset is $\sigma \simeq 15$ Gauss, the vertical lines mark the $3 \sigma$ interval around the mean of signals.

\section{Void-detection algorithm}

To analyze the large number of selected magnetograms we needed an improved and faster version of the void-searching method we used in Paper I. A detailed description of this method has been given by Aikio \& Maehoenen (1998), henceforth AM98, and in the same Paper I. We achieved a remarkable improvement of performances by modifying the most timeconsuming part of the AM98 method: the climbing algorithm. In AM98, each nonmagnetic pixel in the binarized image has to be assigned to its proper void through constructing a continuously connected path "along a monotonically increasing $\nabla D(x)$ " (i.e., the climbing algorithm), $D$ being the distance field generated from the binarized magnetogram.

In the new version of the void-detection algorithm we apply a recursive circle-packing procedure that fills the nonmagnetic area of the binarized magnetogram. The climbing algorithm is then executed only for the centers of the filling circles, which saves a substantial amount of CPU time. The recursive packing (Fig. 2) is achieved by generating a sequence of modified

\footnotetext{
1 See: http://soi.stanford.edu/sssc/progs/mdi/calib. html for more information.
}

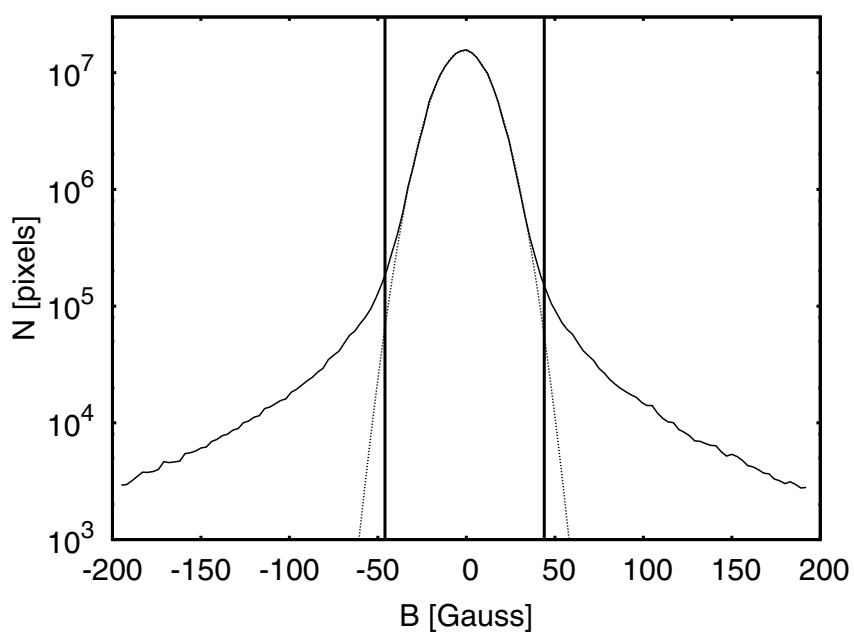

Fig. 1. Magnetic flux density signal from the whole dataset. The Gaussian fit highlights the noise character of the distribution core. The vertical continuous lines mark the $3 \sigma$ interval.

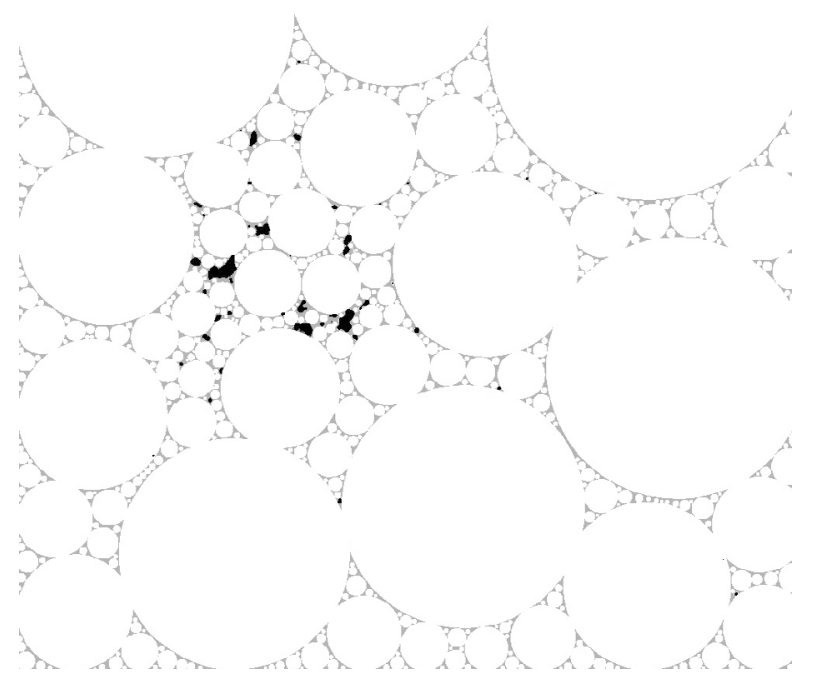

Fig. 2. Circle-packing in a binarized magnetogram: the magnetic structures (dark) are surrounded by circles that cover the nonmagnetic area (gray) almost completely.

distance fields (DFs) relative to the original binarized image. In each step a circle is drawn centered on the maximum of the current DF and with a radius equal to the local DF value (i.e., until it extending the radius until it touches the nearest magnetic structure). In the following step we insert the last generated circle as a fictitious magnetic area to the previously used binarized image and calculate a new DF.

As in the Apollonian circle-packing (Fuchs \& Sauden 2011), a sequence of circles $C_{1}, C_{2}, C_{3}$, etc. with noncrescent diameters that fill the regions empty of magnetic elements is recursively generated. The iteration is stopped when the current $C_{i}$ radius $R$ is smaller than an assigned $R_{\min }$.

With the new algorithm we reduce the time required for the void identification (about 25 times shorter than in AM98).

In Fig. 3 we show an example of the circle-packing coverage relative to the coverage of the magnetogram of our dataset. Panel a shows a the binarized magnetogram. We applied a segmentation fixed threshold of 45 Gauss, which roughly corresponds to $3 \sigma$, to the whole dataset (see Fig. 1). Panel b displays the circle-packing coverage relative to the same magnetogram. 

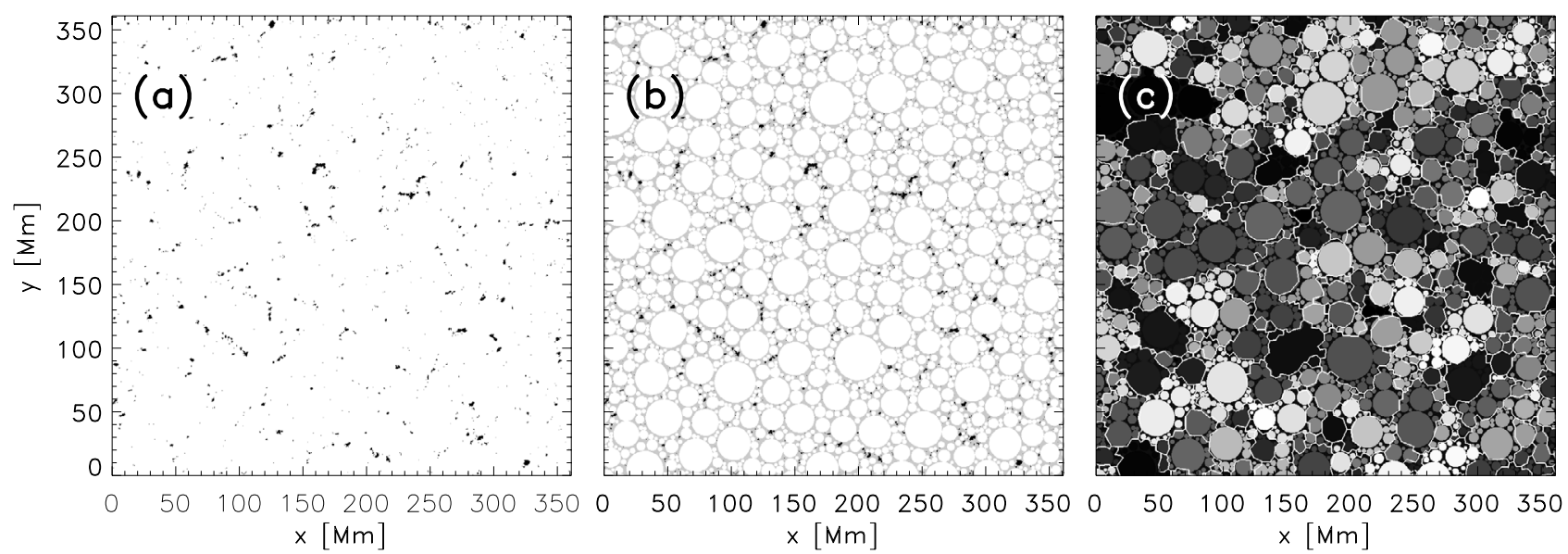

Fig. 3. Panel a) detected magnetic structures (in black) on a typical binarized SOHO/MDI magnetogram. The threshold is equal to $3 \sigma$. Panel b) circles obtained by recursively defining the local DF maxima. Panel c) circles packed together to generate the voids. All the circles pertaining to the same void structure are labeled with the same index, i.e., color (in this figure we highlight voids with a white boundary). The panels show a portion of the MDI FOV for the sake of visualization.

Finally, panel c shows the result of this circle assigning process: all the circles pertaining to the same void structure are labeled with the same index, i.e., color.

It is important to point out that the total area of circles used to identify the voids does not entirely fill the area of a magnetogram. This is due to the cutoff imposed by the smallest circles (in this work we set $R_{\min }=2$ pixel $\simeq 1 \mathrm{Mm}$ ). A careful analysis of a subset of magnetograms, analyzed using both the classical AM98 algorithm and the circle-packing procedure, allowed us to compute the factor $(0.79)$ that corrects for the effect caused by partial coverage of the circles.

\section{Analysis and results}

The void-detection algorithm singled out 252488 voids from the $511 \mathrm{SOHO} / \mathrm{MDI}$ magnetograms. To derive the VSD, we calculated the area $A$ in $\mathrm{Mm}^{2}$ of the observed voids and defined their size as the diameter of a circular region with the same area, size $=2 \sqrt{A / \pi}$.

The VSD is shown in Fig. 4 together with an exponential fit $F=A \times \mathrm{e}^{- \text {Size } / S_{\mathrm{d}}}$, where $S_{\mathrm{d}}$ is the decay constant in $\mathrm{Mm}$. The error bars are calculated as the standard deviation of 1000 resampling ensembles obtained by randomly extracting subsamples of 60 magnetograms from the complete dataset (bootstrapping method). They also highlight the remarkable stability of the VSD during the $\sim 1.5$ years of observations, which, we recall, have been acquired during the period of minimum solar activity between cycles 23 and 24 .

Slightly modifing the threshold at 45 Gauss does not affect the shape of the VSD (see Paper I).

Two important results from the VSD are shown in Fig. 4. First, the computed VSD depends strongly on the size of the voids. Indeed, the distribution agrees very well $\left(R^{2}=0.996\right)$ with an exponential decay with constant $S_{\mathrm{d}}=12.2 \pm 0.2 \mathrm{Mm}$ in the range $10-60 \mathrm{Mm}$. Second, no particular feature is observed around the supergranular $30-50 \mathrm{Mm}$ scale. In other words, the VSD appears to rule out supergranulation as a distinct scale of motion, which supports the multiscale nature of convective motion flows at the solar surface (Nordlund et al. 2009; Yelles Chaouche et al. 2011; Berrilli et al. 2013).

The standard exponential decay satisfactorily fits the distribution, but it also seems to overestimate the size of the largest

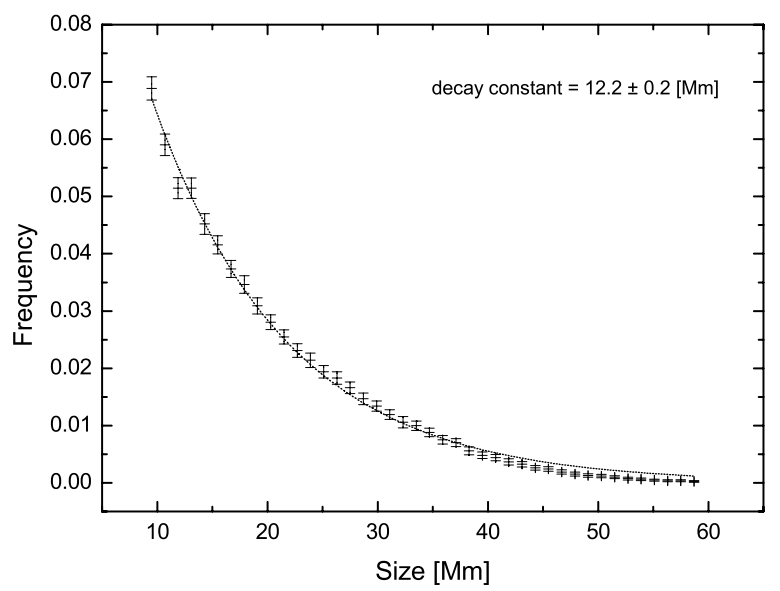

Fig. 4. VSD of 252488 voids singled out in the whole SOHO/MDI dataset. Error bars are computed via a 1000-samples bootstrap on 511 magnetograms. The dotted line represents the exponential decay fit, whose parameters are shown in the upper right corner.

voids. Indeed, a careful analysis shows that there is a regime change around $35 \mathrm{Mm}$. The VSD reveals a departure from a simple exponential decay around $35 \mathrm{Mm}$, in a semilog plot (Fig. 5). To determine whether the departure from the exponential decay is due to projection effects or inhomogeneities on the SOHO/MDI FOV of high-resolution images (about one third of the solar diameter) we checked for a systematic center-to-limb effect in the detection of voids. Figure 6 shows the VSD in four annular regions centered on the FOV center. The radii are chosen to keep each region area constant, and each void is allocated to one of the annular regions according to the position of its baricenter. Baseline offsets are adjusted for each VSD to separate the distributions. We observe a slight trend in the void distributions and the growth of small size voids on moving away from the magnetogram center. Despite this global trend, each of the subsamples still departs from a simple exponential decay around $35 \mathrm{Mm}$. As a consequence, it would seem reasonable to draw the conclusion that projection has a detectable effect on VSD, probably because of the different optical depth of the 


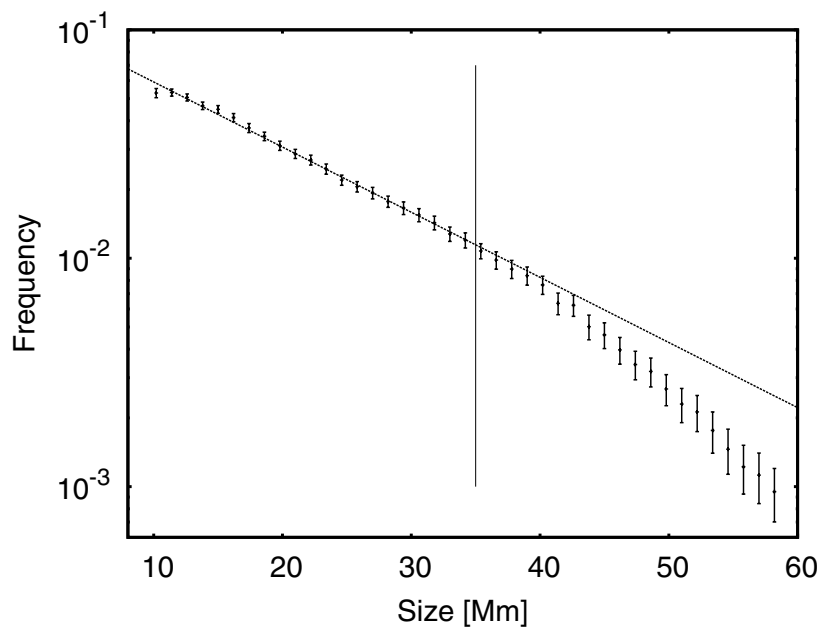

Fig. 5. Symbols with error bars represent the VSD of 252488 voids in semilog scale. Exponential fits in the regions $10-35 \mathrm{Mm}$ and $35-60 \mathrm{Mm}$ are represented by dotted lines. In the range $35-60 \mathrm{Mm}$ the decay constant is $S_{\mathrm{d}}=7.6 \pm 0.2 \mathrm{Mm}$, while in the range $10-35 \mathrm{Mm}$ the value is $S_{\mathrm{d}}=12.4 \pm 0.2 \mathrm{Mm}$. The continuous vertical line marks the $35 \mathrm{Mm}$ scale.

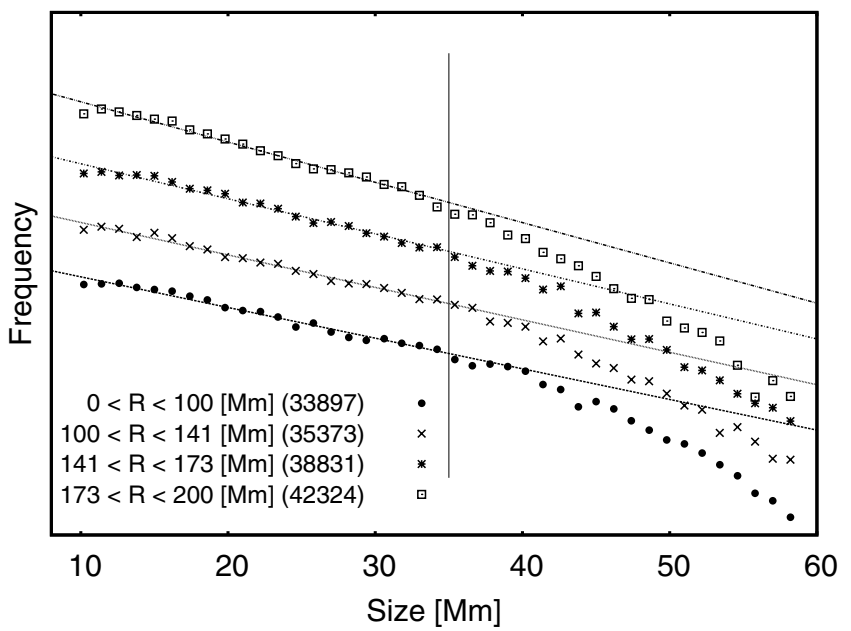

Fig. 6. VSDs for subsets of voids extracted in four annuli centered on the solar disk center. The distributions corresponding to the four regions are represented by the different lines. Each of the subsets retains the regime modification at about $35 \mathrm{Mm}$ indicated by the vertical line. The number between brackets indicates the number of voids found in each annulum. Baseline offsets are adjusted for each VSD to separate the distributions.

designed regions, but the regime modification around $35 \mathrm{Mm}$ is a real feature of solar (turbulent) convection.

A basic further question about the sparse pattern of magnetic structures, as singled out by the thresholding in our method (Fig. 3a), is whether such structures are distributed in a random fashion or if they are somehow ordered. The same question can be expressed in terms of VSD: is it possible to obtain a similar distribution from a randomly distributed pattern of magnetic structures?

We investigated this possibility by applying the voiddetection algorithm on shuffled images. These images were obtained by shuffling the magnetic structures of each binarized magnetogram in the FOV. This reshaping was performed by avoiding the superimposition of the translated structures, that is, by preserving the total number of magnetic pixels in each
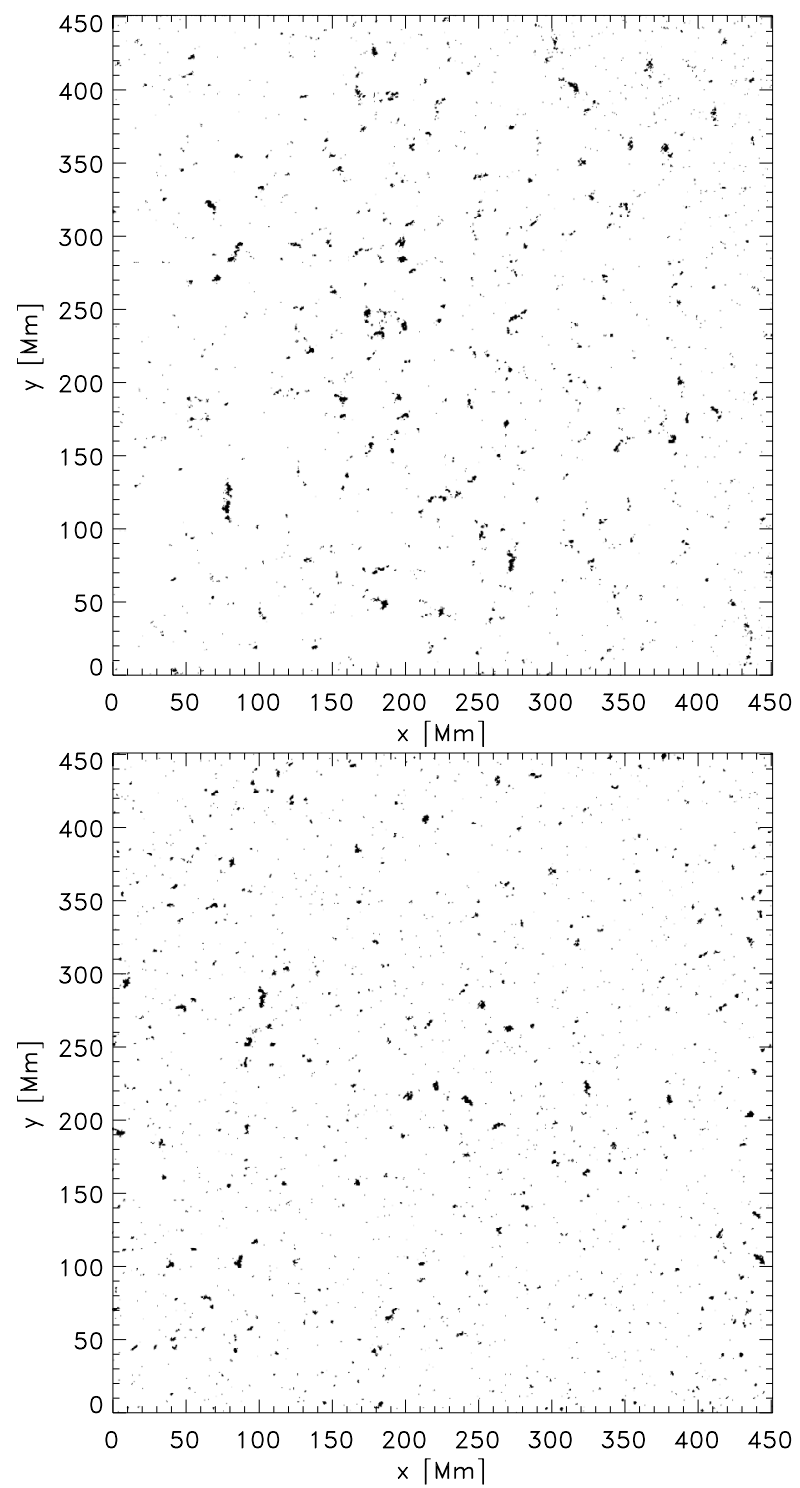

Fig. 7. Upper panel: MDI magnetogram (July 11, 2008). Lower panel: results after shuffling the original magnetic structures.

binarized image. The original and shuffled images corresponding to the MDI magnetogram acquired on July 11, 2008 are shown for comparison in Fig. 7.

The resulting VSDs are shown in the semilog plot of Fig. 8 (crosses) together with the original VSD (symbols with errorbars). In this case, the shuffling was applied to the images of the second half of 2008 (167 magnetograms). In the same figure, a Gaussian fit of the VSDs is shown. The evident difference between the two distributions strongly suggests that the VSD obtained from SOHO/MDI magnetograms is not compatible with a simple random-like pattern as discussed in Consolini et al. (2003), Berrilli et al. (2004), Hirzberger et al. (2008).

\section{Conclusions}

We analyzed the distribution of void sizes in quiet-Sun magnetograms to investigate the multiscale magnetic pattern observed in the photosphere, which means the organization scales of convection (Yelles Chaouche et al. 2011), in the 10-60 Mm range. In particular, we investigated the geometrical properties of the voids of magnetic field, associated with the boundaries of 


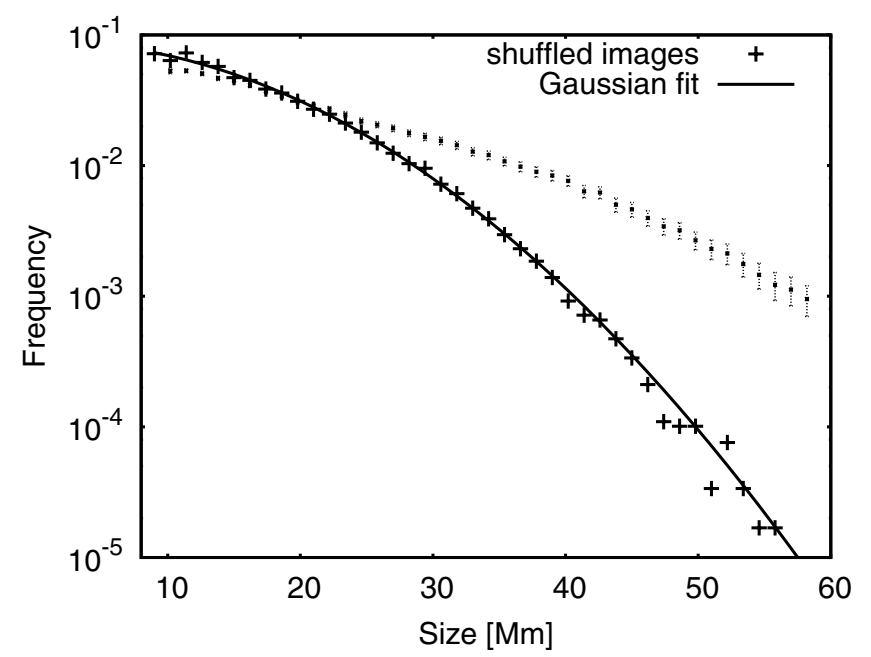

Fig. 8. Symbols with error bars represent the void size distribution of 252488 voids (as in Fig. 4) in semilog scale. Crosses show the mean VSD computed by shuffling the magnetic structures in each magnetogram. The Gaussian fit is represented by the continuous curve.

large-scale cells of overturning plasma using 511 SOHO/MDI high-resolution magnetograms acquired during the unusually long solar activity minimum between cycles 23 and 24 . We selected this period to minimize the influence of active regions on the spacial pattern through convective motions. Our study was based on a fast void-detection procedure that uses a circlepacking algorithm to obtain robust statistics.

During the 1.5 years covered by the dataset the computed VSD showed a remarkable stability. There is a slight dependence of the VSD on the curvature of the FOV, that is, on the position of voids with respect to the angle between the line of sight and the solar surface vertical. It is evident that this angle interferes with the magnetogram segmentation with a fixed and constant threshold. Because of the magnitude of the effect that we observed in our dataset, it was beyond the scope of this work to deal with any segmentation improvement, for the moment. The observed stability has the potential to be used as a quantitative definition of what constitues a "quiet Sun" as well as "solar activity minimum".

We have established that the VSD shows a quasi-exponential decay in the observed range. The monotonic distribution and the lack of marked features in the $10-60 \mathrm{Mm}$ range point out the absence of a supergranular scale and support the multiscale hypothesis of convective motion flows at the solar surface (Nordlund et al. 2009; Yelles Chaouche et al. 2011; Berrilli et al. 2013): but it does not rule out the reality of convection structures with these sizes. For example, an advective-interaction model (Rast 2003) is able to explain the presence of flow at large scales as the result of a local merger of downward plumes.

In addition to the quasi-exponential decay, we found that the void distribution departs from a simple exponential decay at about $35 \mathrm{Mm}$.

We speculated about a possible scenario that leads to a convective instability scale. Instabilities in the convection boundary layer located in the photosphere could be induced when the distance between downward plumes exceeds the above-mentioned critical value of $35 \mathrm{Mm}$. At this supergranular scale buoyancy forces become sufficient to trigger the formation of a new downflow plume with an instability mechanism similar to that reported in Rast (1995) for the granulation scale. This mechanism would be able to introduce thermal plumes into the stratified compressible plasma which could provide possible sites for magnetic field concentrations. These new magnetic regions could limit the maximum size of voids and reduce the number of observed voids with typical sizes greater than $35 \mathrm{Mm}$.

Acknowledgements. We thank Stuart Jefferies and Juri Toomre for the stimulating discussions. SoHO is a mission of international cooperation between ESA and NASA. This work was supported in part by the EC Home Affairs CIPS program SPARC: Space Awareness for Critical Infrastructure research grant. This research work is partly supported by the Italian MIUR-PRIN grant 2012P2HRCR on "The active Sun and its effects on Space and Earth climate" and by the Space Weather Italian COmmunity (SWICO) Research Program.

\section{References}

Aikio, J., \& Maehoenen, P. 1998, ApJ, 497, 534

Berrilli, F., Florio, A., \& Ermolli, I. 1998, Sol. Phys., 180, 29

Berrilli, F., Del Moro, D., Consolini, G., et al. 2004, Sol. Phys., 221, 33

Berrilli, F., Del Moro, D., Russo, S., Consolini, G., \& Straus, T. 2005, ApJ, 632, 677

Berrilli, F., Scardigli, S., \& Giordano, S. 2013, Sol. Phys., 282, 379

Consolini, G., Berrilli, F., Florio, A., Pietropaolo, E., \& Smaldone, L. A. 2003, A\&A, 402, 1115

Criscuoli, S., Ermolli, I., Del Moro, D., et al. 2011, ApJ, 728, 92

Crouch, A. D., Charbonneau, P., \& Thibault, K. 2007, ApJ, 662, 715

Del Moro, D., Berrilli, F., Duvall, T. L., Jr., \& Kosovichev, A. G. 2004, Sol. Phys., 221, 23

Del Moro, D., Giordano, S., \& Berrilli, F. 2007, A\&A, 472, 599

DeRosa, M., \& Toomre, J. 2004, ApJ, 616, 1242

Duvall, T. L., Jr., \& Birch, A. C. 2010, ApJ, 725, L47

Fuchs, E., \& Sauden, K. 2011, Exp. Mathematics, 20, 380

Giannattasio, F., Stangalini, M., Berrilli, F., Del Moro, D., \& Bellot Rubio, L. 2014, ApJ, 788, 137

Goldbaum, N., Rast, M. P., Ermolli, I., Sands, J. S., \& Berrilli, F. 2009, ApJ, 707, 67

Hirzberger, J., Gizon, L., Solanki, S., \& Duvall, T. L. 2008, Sol. Phys., 251, 417 Krivova, N. A., \& Solanki, S. K. 2004, A\&A, 417, 1125

Leighton, R. B. 1964, ApJ, 140, 1547

Leighton, R. B., Noyes, R. W., \& Simon, G. W. 1962, ApJ, 135, 474

Liu, Y., \& Norton, A. A. 2001, SOI-Technical Note 01-144

Lisle, J., De Rosa, M., \& Toomre, J. 2000, Sol. Phys., 197, 21

Lisle, J. P., Rast, M. P., \& Toomre, J. 2004, ApJ, 608, 1167

Lites, B. W., Kubo, M., Socas-Navarro, H., et al. 2008, ApJ, 672, 1237

Martìnez González, M., Manso Sainz, R., Asensio Ramos, A., \& Hijano, E. 2004, ApJ, 755, 175

Meunier, N., Roudier, T., \& Rieutord, M. 2008, A\&A, 488, 1109

Nordlund, A., Stein, R. F., \& Asplund, M. 2009, Liv. Rev. Sol. Phys., 6, 2

Orozco Suárez, D., \& Bellot Rubio, L. R. 2012, ApJ, 751, 2

Orozco Suárez, D., Katsukawa, Y., \& Bellot Rubio, L. R. 2012, ApJ, 758, L38

Rast, M. P. 1995, ApJ, 443, 863

Rast, M. P. 2003, ApJ, 597, 1200

Rieutord, M., \& Rincon, F. 2010, Liv. Rev. Sol. Phys., 7, 2

Rieutord, M., Roudier, T., Malherbe, J. M., \& Rincon, F. 2000, A\&A, 357, 1063

Sánchez Almeida, J. 2003, A\&A, 411, 615

Scherrer, P. H., Bogart, R. S., Bush, R. I., et al. 1995, Sol. Phys., 162, 129

Schrijver, C. J., Hagenaar, H. J., \& Title, A. M. 1997, ApJ, 475, 328

Simon G. W., \& Leighton R. B. 1964, ApJ, 140, 1120

Stein, R. F., Benson, D., Georgobiani, D., Nordlund, A., \& Schaffenberger, W. 2007, Unsolved Problems in Stellar Physics: A Conference in Honor of Douglas Gough, 948, 111

Yelles Chaouche, L., Moreno-Insertis, F., Martínez Pillet, V., et al. 2011, ApJ, 727, L30 